\title{
USO DE TIC EN ESCUELAS PÚBLICAS DE ECUADOR: ANÁLISIS, REFLEXIONES Y VALORACIONES
}

\author{
ICT USE IN PUBLIC SCHOOLS OF ECUADOR: ANALYSIS, REFLECTIONS \\ AND REVIEWS
}

\author{
Mónica Peñaherrera León \\ mpleon@ujaen.es
}

Universidad de Jaén

\section{RESUMEN}

El artículo presenta los datos de una investigación centrada en analizar los efectos de la implementación del Proyecto "Mejoramiento de la Calidad de la Educación Pública para el fortalecimiento del Aprendizaje a través de las TIC: De tal palo, tal astilla", en las escuelas públicas de Ecuador.

Para la recogida de datos hemos utilizado las siguientes técnicas cualitativas: entrevistas, observaciones de clases y análisis documental. Los resultados indican que las TIC modifican las estructuras de los centros escolares a nivel organizativo, sin que altere sustancialmente las prácticas educativas del aula. No obstante, el uso de las TIC fomenta en el alumnado una alta motivación para aprender.

PALABRAS CLAVE: TIC, proceso de enseñanza-aprendizaje, innovación educativa.

\section{ABSTRACT}

This article presents the research data focused on analyzing the effects of a project" Improving the Quality of Public Education for the strengthening of Learning through ICT: Like father, like son" in the public schools of Ecuador.

For the collection we used different qualitative techniques: interviews, classroom observations and document analysis. The results indicate that ICTs alter the tructures of schools at the organizational level, without substantially alteringclassroom teaching practices. Finally, of note is the high motivation that students have to learn through ICT.

KEY WORDS: ICT, teaching-learning process, educational innovation. 


\section{INTRODUCCIÓN}

En otro lugar ${ }^{1}$, habíamos descrito el avance que América Latina está teniendo es los últimos años con respecto a la incorporación de las TIC en distintas ámbitos, desde el gubernamental, con la implantación de los e-gobiernos, hasta en la educación, con la incorporación del uso de ordenadores personales por alumno ${ }^{2}$.

Las políticas públicas en relación a las TIC en los países de América Latina se iniciaron hacia mediados de los años noventa. El fenómeno de la globalización impulsó fuertemente la atención de los gobiernos en las TIC.

A inicios del año 2000 algunos países iberoamericanos comenzaron a dar sus primeros intentos de diseñar una política pública acorde con la llamada "Sociedad de la información". Estos intentos fueron más tarde reforzados con las dos Cumbres Mundiales para la Sociedad de la Información (CMSI) en los años 2003 y 2005 respectivamente, y la inclusión de las TIC dentro de los Objetivos de Desarrollo del Milenio de las Naciones Unidas (ODM), que incorporaban esta misma visión (ONU, 2000).

Ecuador no es ajeno a las tendencias de la Sociedad de la Información, está dotando a las escuelas públicas de equipamiento de aulas de informática con acceso a internet y con miras al modelo tecnológico 1x1 (Peñaherrera, 2011 a). En el 2010, según expresa el informe "Rendición de Cuentas" del Ministerio de Educación del Ecuador, no utilizar las TIC es "no vivir el progreso"

Aunque, la primera iniciativa oficial destinada a incorporar las TIC en el sistema educativo comenzó en el 2002 cuando se dota a los maestros de un PC y se implementa un programa de capacitación destinado al uso pedagógico del ordenador, se trata del programa Maestr@s.com. Después de aquella iniciativa hubo un cierto estancamiento, unido sobre todo a los cambios de gobiernos en tan pocos años.

En el 2006 es cuando Ecuador formalmente se afianza en incorporar las TIC a los gestión pública y a los procesos educacionales a través del Libro Blanco de la Sociedad de la Información, como un instrumento que recoge los planteamientos de diversos sectores del Estado y que puede constituir el marco de la política de TIC para los próximos años (CONATEL, 2006).

La integración de las TIC en el sector educativo del país andino ha apuntado a la dotación de infraestructuras, equipamiento de aulas con ordenadores y recursos informáticos, dotación de software educativo, capacitación al profesorado, creación de portales educativos, soporte técnico a las escuelas, entre otros.

Recientemente, Ecuador ha elaborado su primer documento base de estándares TIC en concordancia con la propuesta de estándares educativos que se lleva a cabo actualmente (Ministerio de Educación de Ecuador, 2012).

\footnotetext{
${ }^{1}$ Peñaherrera, M. (2011). Evaluación de un programa de fortalecimiento del aprendizaje basado en el uso de las TIC en el contexto ecuatoriano. Revista Iberoamericana de Evaluación http://www.rinace.net/riee/numeros/vol4-num2/art4.pdf. 14 enero 2012.

${ }^{2}$ Ciertas iniciativas están orientadas al modelo 1x1, por ejemplo, en Uruguay (El Plan Ceibal), Perú (Laptop por niño), Argentina (Conectar Igualdad), Chile (uso de tecnología portátil 1 a 1 en el aula) y en Ecuador (Mi Compu). (Lagos y Quiroz, 2011).
} 
Las TIC vinculadas al sector educativo es un ámbito de creciente interés dentro de las políticas públicas y una necesidad en estos últimos años en el país andino.

\section{EL PROYECTO “DE TAL PALO, TAL ASTILLA”}

Actualmente, "De tal palo tal astilla" ${ }^{3}$, es un proyecto que busca resolver de manera integral la baja calidad de la educación con el apoyo de las TIC.

El proyecto se articula en cuatro estrategias (ESPOL- CTI, 2007): capacitación y seguimiento, desarrollo de infraestructura física y tecnológica, desarrollo, actualización y adaptación curricular, y gestión y mejoramiento continuo de la educación:

- La primera de ellas, capacitación y seguimiento, tiene como fin dotar a los maestros de habilidades pedagógicas y tecnológicas, para que sean desarrolladas de manera efectiva en el aula. Así también, ha contemplado capacitar a los directivos de los centros educativos, para la gestión, liderazgo y administración educativa.

- La segunda estrategia, desarrollo de infraestructura física y tecnológica, busca, a través de la modernización de aulas y la inclusión de tecnología de punta, dotar a estudiantes y docentes de herramientas que les permiten acceder a información universal e interactuar con pares en otros lugares del mundo. El desarrollo de habilidades tecnológicas se fundamenta en los ambientes de informática educativa basados en herramientas para pensar como: Scratch y la Robótica Mind Storms, así como la introducción de una filosofía educacional y práctica constructivista asociada con el uso de estos ambientes informáticos.

- La tercera es la del desarrollo, actualización y adaptación de contenidos programáticos, articulando las ciencias con énfasis en valores y la identidad provincial de orenses de acuerdo a la reforma escolar. De manera que los contenidos contribuyan a formar ciudadanos preparados para aprender durante toda la vida.

- Finalmente, la cuarta estrategia, gestión y mejoramiento continuo de la educación, busca institucionalizar y provincializar la evaluación, rendimiento de cuentas y mejoramiento continuo de la educación orense, con lo cual se genera una cultura de calidad en las instituciones educativas y se garantiza la sostenibilidad de las actividades vinculadas a este proyecto a lo largo del tiempo.

El proyecto abarca los Cantones Huaquillas y Arenillas de la Provincia del Oro, incluyendo las zonas rurales y áreas urbano - marginales.

El proyecto ha buscado en un período de tres años implementar estas estrategias de forma que al finalizar la ejecución del proyecto, la calidad de la educación de la Provincia sea alta.

\footnotetext{
3 Para más información del proyecto “De tal palo tal astilla" se recomienda consultar el portal web del mismo en la siguiente dirección: www.cti.espol.edu.ec
} 
El estudio que presentamos se encuadra en el marco de este proyecto. El propósito que nos ha llevado a planificar y desarrollar esta investigación se fundamenta desde el planteamiento realizado por Area, (2005:4 a):

"Tenemos mucha información empírica sobre las TIC en las escuelas, pero nos falta construir una teoría sobre este fenómeno particular de la realidad escolar que nos permita comprender qué sucede cuando los ordenadores entran en las escuelas, las causas de la resistencia del profesorado a integrar estas tecnologías en su práctica docente, o cómo implementar exitosamente estrategias de incorporación escolar de las TIC en un determinado contexto".

La pregunta obligada es si gracias a la introducción de las TIC se está propiciando un cambio profundo en las prácticas y paradigmas educativos prevalecientes, en la forma y contenido de lo que se aprende, se enseña y se evalúa. Este es la razón que nos ha llevado a realizar esta investigación en el contexto de Iberoamérica, dónde las TIC están emergiendo con mayor fuerza.

\section{METODOLOGIA}

\section{Objetivo}

Con este proyecto de investigación hemos pretendido analizar y evaluar el proceso de integración pedagógica de las nuevas tecnologías de la información y comunicación en las prácticas de enseñanza y aprendizaje de centros de educación primaria participantes en el proyecto "De Tal Palo, Tal Astilla". Concretamente, las cuestiones generales que han guiado la planificación y desarrollo del estudio fueron:

- ¿Qué uso hace el profesorado y el alumnado de las tecnologías digitales?

- ¿Qué impacto tiene las TIC sobre la enseñanza y aprendizaje desarrollado en las aulas?

- ¿Qué innovaciones introduce el uso de las nuevas tecnologías en:

- la organización del centro escolar,

- en la enseñanza en el aula,

- en el desarrollo profesional del docente y,

- en el aprendizaje del alumnado

A partir de estas cuestiones, la investigación plantea las siguientes dimensiones:

\section{Dimensión 1: Organización del centro}

Cada vez, va siendo más usual la utilización de los medios en las tareas de administración y gestión del centro, sobre todo del medio informático. De ahí, que la irrupción de las TIC provocan cambios en las organizaciones educativas, producen un aumento de las interrelaciones entre los diferentes miembros de la comunidad ofreciendo la oportunidad de una comunicación más fluida entre éstos.

Aunque, ello por sí mismo no es suficiente. Tal y como apunta Kagel (2003: 279), "es necesario pensar el cómo se las implementa y qué idea subyace en esa implementación". 
Este último planteamiento nos hace pensar que no existe una única forma de organizar los medios en las instituciones educativas, pues ello dependerá de una serie de variables que irán desde la política del centro, el modelo organizativo, la cultura escolar en la que se desenvuelva, el nivel educativo, o la diversidad de tecnologías que puedan ponerse en funcionamiento.

Por otro lado, en distintos trabajos se han intentado sistematizar los factores y procesos de integración de las TIC en el medio escolar. Los resultados de los estudios coinciden que la incorporación de los ordenadores en el aula es un proceso complejo, sometido a distintos niveles e instancias de naturaleza política, empresarial, social y pedagógica (Area, 2010 b). De forma que la integración de las TIC dependerá más o menos de la política y cultura organizacional, del nivel técnico, directivo o profesional y el enfoque que se le otorgue.

\section{Dimensión 2: Desarrollo profesional}

Está claro que las TIC involucra no sólo los "haceres" sino también los "saberes" en los docentes. Las TIC sirven como herramientas para aprehender esos "saberes" lo largo de la vida. Al respecto, Adell (2011) señala lo siguiente:

- Los docentes deben aprender activamente y no esperar a que alguien les enseñe.

- Formar parte de redes sociales profesionales.

- Participar de proyectos colectivos.

Las TIC son herramientas de apoyo en el desarrollo profesional de los docentes, entre otras razones, porque les permiten aprender en forma práctica a utilizar la tecnología (UNESCO: 2004).

Salomé (2010) en una investigación realizada sobre el uso de las TIC en docentes ecuatorianos, encontró que las TIC la consideran como un recurso importante para perfeccionarse en forma continúa y lograr así un desarrollo profesional permanente (81\%).

El objetivo se consigue con un plan de formación eficazmente articulado con las necesidades y expectativas de los docentes en relación al uso de las TIC, en el que se implique participativamente para la adquisición de competencias digitales. Es importante señalar, que no sólo la formación es importante, sino también la autoformación.

\section{Dimensión 3: Prácticas de enseñanza}

Con la irrupción de las TIC en el aula las prácticas de enseñanza se ven alteradas, sobre todo, por las posibilidades que abren las nuevas tecnologías. Algunos estudios han observado que en los lugares donde las TIC se transforman en una parte integral de la experiencia en la sala de clases, hay mayores evidencias de impactos en el aprendizaje y el desempeño de los estudiantes (Condie y Munro, 2007). Sin embargo, ello no depende sólo de la tecnología sino también de las capacidades, actitudes y creencias pedagógicas de los profesores.

Por ejemplo, un estudio con una muestra nacional de profesores desde 4to básico en adelante en Estados Unidos mostró que junto con ciertas condiciones mínimas de infraestructura y capacitación técnica, la filosofía pedagógica de los profesores de asignaturas estaba relacionado con el uso o no uso de las TIC en la sala de clases (Becker, 2000). Se encontró que profesores que tenían una visión pedagógica constructivista -que en contraste con una visión pedagógica transmisiva o tradicional, se caracteriza por 
conceptualizar el aprendizaje de una persona como el resultado de integrar nuevas ideas y argumentos a las propias creencias y conceptos y darle por lo tanto al estudiante un rol más activo en el aprendizaje-, eran más proclives a usar las TIC durante sus clases.

Autores como Cox y Webb (2004), en un estudio acerca de las ideas, creencias y acciones sobre las TIC de los profesores, han identificado un rango de actividades que se relacionaba con su uso en las prácticas de enseñanza, estableciendo las siguientes: los tipos de recursos TIC que los profesores escogen usar; su conocimiento de la propia asignatura y del potencial de las TIC para reforzar el aprendizaje específico en ella; y su habilidad para integrar las TIC en su programa curricular completo. La evidencia recopilada por estos investigadores muestra que cuando los profesores usaban su conocimiento tanto de la asignatura como de la forma como los estudiantes entendían la asignatura, su uso de las TIC tenía un efecto más directo en el logro del estudiante.

Por su parte, Cabero y Llorente (2010) apuntan que con el uso de las TIC, los docentes deben atender más a la creación de conocimientos que la reproducción de los mismos. Debe por tanto tender hacia una escuela 2.0, que sería aquella que teniendo en cuenta las nuevas características de los alumnos y las posibilidades de las nuevas herramientas de comunicación que se han originado en la red, se plantea una nueva forma de actuar y de replantear el hecho educativo.

A grandes rasgos, supone pasar de centrarnos en la transmisión de información a la construcción del conocimiento. Por tanto, la idea de indagar esta cuestión es fundamental para desentrañar cómo y de qué manera alteran o no las TIC las prácticas de enseñanza.

\section{Dimensión 4: Aprendizaje}

Uno de los hallazgos más consistentes es el impacto de las TIC en la motivación y la concentración del alumno (Claro, 2010). Un estudio realizado por Passey y Foreim (2004), destaca que las TIC ayudaban a los estudiantes a tener tipos más positivos de motivación para el aprendizaje y podían ofrecer medios a través de los cuales los estudiantes podían visualizar éxito. Adicionalmente los profesores sentían que las TIC tenían un impacto positivo en el interés y actitudes de los estudiantes con el trabajo escolar.

Otros estudios realizados arrojan también algunos resultados relativos al desarrollo de destrezas transversales, tales como comunicación, colaboración, aprendizaje autónomo, y trabajo en equipo. Así también, habilidades cognitivas de orden superior, tales como pensamiento crítico, la resolución de problemas, la capacidad de análisis y la creatividad (Condie y Munro, 2007; Balanksat, 2006; Cox, 2003; McFarlane, 2000; Peñaherrera, 2008 b).

\section{La selección de casos}

El estudio sobre el que se basa este artículo, se realizó en tres escuelas primarias públicas de la Provincia del Oro. Seleccionadas por considerarse una de las veinticinco escuelas "punta" de esta categoría, en lo que a la introducción de las TIC se refiere, tanto por su dotación informática como por

considerarse centros que tienen interés en la integración de las TIC en el aula.

Esto significa que en el momento de llevarse a cabo la investigación, los centros escolares habían desarrollado múltiples proyectos con el programa Scratch. 
Por tanto, se constituyeron casos representativos, "típicos" (Goetz \& LeCompte, 1988) de los centros que promueven la enseñanza asistida por ordenador.

Otro criterio utilizado ha sido la accesibilidad y disponibilidad de los centros educativos para levantar la información.

El estudio se centró en el segundo ciclo ( 3 ㅇ y 4 o curso) en el área de Lenguaje y Comunicación. De las cuatro horas semanales de Lenguaje que corresponden a esta etapa, una a la semana se desarrollaba en el aula de informática, es decir, se dedicaba a la utilización de algún programa informático.

El núcleo central del análisis de los datos para alcanzar los objetivos de la investigación se centró, en primer lugar, en el análisis de los soportes lógicos utilizados, y en segundo lugar, en el de las interacciones que tenían lugar en las clases.

En este artículo se presenta solamente el análisis de las aplicaciones informáticas utilizadas y las interacciones suscitadas entre los niños y niñas de la clase a partir del programa informático Scratch.

El programa Scratch ${ }^{4}$ es un lenguaje de programación multimedia que permite crear animaciones interactivas, cuentos, juegos, y compartir sus creaciones en la página web. Es un entorno donde los niños y jóvenes pueden expresar sus ideas, mediante actividades creativas y lúdicas.

Una de las ventajas importantes que tiene es la sencillez del entorno que ayudar a desarrollar las capacidades de resolución de problemas. Además inicia a los niños en una lógica de un lenguaje de programación básico, desarrollando su creatividad, capacidad de análisis, interpretación, síntesis y argumentación, con lo que se estimula y pone en marcha un pensamiento lógico y estructurado. En consecuencia es una excelente herramienta para la escuela, pudiendo a través de ella desarrollar procesos transversales de aprendizaje.

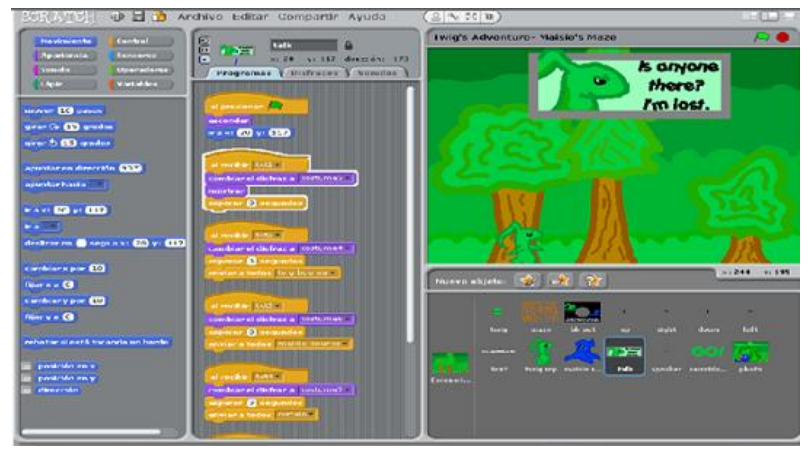

Fig. 1. Tomado de http://scratch.mit.edu/projects/UnprovenTheorem/2220471

\section{Técnica de recogida de datos}

Las técnicas utilizadas para recoger la información relativa a cada una de las dimensiones del estudio, se consideran en la tabla 1:

\footnotetext{
${ }^{4}$ Este ambiente de aprendizaje está siendo desarrollado por el grupo de investigación Lifelong Kindergarten en el Laboratorio de Multimedia del Massachusetts Institute of Technology (MIT), en colaboración con el grupo de investigación de la Escuela de estudio de educación e información de la Universidad de California en los Ángeles (UCLA). Veáse. http://scratch.mit.edu
} 


\begin{tabular}{|c|c|}
\hline Dimensión de análisis & Técnica de recogida de datos \\
\hline Organización y gestión TIC. & $\begin{array}{l}\text { - Entrevistas directores. } \\
\text { - Entrevista coordinador TIC local. } \\
\text { - Entrevista asesor TIC. } \\
\text { - Recogida y análisis documental. } \\
\text { - Observaciones. }\end{array}$ \\
\hline $\begin{array}{l}\text { Enseñanza } \quad \text {-Aprendizaje } \\
\text { con TIC. }\end{array}$ & $\begin{array}{l}\text { - Entrevistas a docentes. } \\
\text { - Entrevistas coordinador TIC local. } \\
\text { - Observaciones de aula. }\end{array}$ \\
\hline $\begin{array}{l}\text { Desarrollo profesional } \\
\text { docente. }\end{array}$ & $\begin{array}{l}\text { - Entrevistas equipo directivo. } \\
\text { - Entrevista coordinador TIC. } \\
\text { - Entrevista asesor TIC. } \\
\text { - Entrevistas a diversos docentes. }\end{array}$ \\
\hline
\end{tabular}

Tabla 1. Dimensiones y técnicas de recogida de información

\section{Análisis de recogida de datos}

Hemos utilizado, el enfoque de análisis de cruce de casos (Cross-case-analysis), se trata de hacer primero un análisis dentro de cada caso y luego un análisis transversal de los demás casos (Merriam, 1988, 1998; Yin, 1994; Huberman y Miles 1994; Patton, 1990). El estudio de casos múltiples hace posible la construcción de una cadena lógica de evidencia (Yin, 1994; Miles y Huberman, 1994). En otras palabras, se utiliza el análisis cruzado de casos para buscar una cadena de evidencias de las relaciones estudiadas sobre la base de la estructura.

En la organización de los datos, Patton (1990) identifica dos fuentes principales: 1) las preguntas que se generan durante la fase conceptual del estudio y 2) el análisis, la comprensión, y las interpretaciones que surgieron durante la recolección de datos (p. 378). Tomando estos criterios, hemos organizado nuestros datos.

Además, una revisión de la bibliografía utilizada en el análisis de datos ayudó a clarificar y obtener una comprensión más profunda de lo que se relacionó con diferencia de las categorías que se repiten.

Las transcripciones de entrevistas, notas de observaciones de campo, y de los documentos se realizaron mediante la codificación y la comparación, las categorías más comunes que respondieron a las preguntas de investigación fueron identificadas. A continuación, se cotejaron los resultados y se organizaron los datos en una matriz para el análisis cruzado de casos.

\section{RESULTADOS}

A continuación ofrecemos una síntesis de los resultados generales obtenidos en los estudios de caso realizados.

\section{Dimensión 1: Organización y gestión de las TIC en el centro escolar}


La organización y gestión de las TIC en el proyecto "De tal Palo, tal Astilla", está conformado por un Comité Institucional. El cual fue elegido de manera participativa por la comunidad educativa de la Provincia de El Oro (Dirección Provincial de Educación, Ayuntamiento, Universidades, escuelas, empresas).

- Está también conformado por nivel ejecutivo, integrado por un conjunto de técnicos designados por la ESPOL más el equipo de soporte y logística. Este nivel es el responsable operativo del cumplimiento de los objetivos y metas del proyecto.

- El proyecto ha entregado a cada centro escolar un aula específica con infraestructura tecnológica que está compuesta de 12 a 15 ordenadores, dotados del siguiente software: Scratch y la Robótica - Mind Storms, impresora, cañón multimedia, una pantalla de proyección. Estos ordenadores tienen acceso a internet

- La estrategia para solventar los problemas técnicos es gestionada por un servicio técnico del CTI. Se han construido 2 laboratorios móviles (contenedor) para atender escuelas de la provincia con dificultades para acceder a la energía eléctrica e Internet. Cada laboratorio móvil cuenta con 8 ordenadores, aire acondicionado, impresora, mobiliario para laboratorio, paneles solares. Los laboratorios hacen un recorrido permanente por las escuelas que necesitan el apoyo técnico.

Por otro lado, existe adicionalmente un equipo técnico de la zona que hace seguimiento a los problemas técnicos que surjan.

- En el proyecto existe la figura de "Facilitadores locales TIC" tanto para el ámbito de la Pedagogía, como para la Tecnología. El perfil que tienen atiende a uno y otro ámbito. Estas personas no pertenecen a la plantilla docente. Las principales funciones son las de apoyar a los profesores en la elaboración de proyectos de aula, motivar al profesorado, facilitar recursos y en general, asistir al profesorado tanto de la parte pedagógica como tecnológica.

- La figura de "Facilitadores locales TIC" local constituye un elemento fundamental en los centros para la promoción de la integración de las TIC en el ámbito escolar. Creemos que si esta coordinación es débil o fallara, existe probabilidades de que el proyecto "De tal palo, tal astilla" apenas tenga incidencia real sobre la vida misma de los procesos educativos, de ahí la importancia de crear capacidad local para la sosteniblidadad del proyecto.

- Podemos indicar también, en lo que a esta dimensión se refiere, la implicación que las escuelas han tenido se enfoca sólo a la adecuación y a la mantención del aula de informática. Sin embargo, no hemos observado que los propios centros educativos dispongan de un protocolo de organización técnica y gestión de las TIC.

- No obstante, es el propio proyecto "De tal palo, tal astilla", el que contempla entre sus procedimientos, el seguimiento para solventar los problemas técnicos.

- En nuestras observaciones realizadas, hemos de comentar que los directores de los centros facilitan la implementación del proyecto en el centro escolar, esto es, la adecuación y mantenimiento de las aulas de informáticas, la asistencia a la formación, etc. No obstante, la gestión sólo queda hasta ahí, en adelante su implicación es poca o nula. 


\section{Dimensión 2: Desarrollo profesional docente}

- El profesorado ha tenido una formación continua en temáticas relacionadas a la tecnología y pedagogía. Cerca del $60 \%$ de la capacitación apunta al uso de las TIC con enfoque pedagógico, y el porcentaje restante (40\%) hasta ahora, se vincula al área de tecnología.

- Los temas de aspectos pedagógicos han cubierto los siguientes ámbitos: educación inicial, administración y gestión educativa, incorporación de las TIC en el aula desde la pedagogía, programación didáctica, etc.

- Los profesores reciben una certificación por los cursos aprobados y estos son reconocidos por la administración educativa de la Provincia de El Oro y sirven para méritos académicos.

- El profesorado recibe también formación en lengua extranjera, concretamente en inglés. El proyecto considera un valor añadido el conocimiento de esta lengua para ampliar búsqueda de información, conocimiento, otras experiencias y contactos en la red.

- Hemos de decir, que la capacitación beneficia a los directores de los centros educativos con el propósito de que tengan el mismo conocimiento que los demás profesores y de esta manera apoyen la incorporación de las TIC y garanticen una política institucional coherente.

- En uno de los casos investigados, un profesor comenta sobre este tema lo siguiente:

"Nos íbamos a dar cuenta de que íbamos a estar capacitando, innovando, teníamos que asistir a cursos o seminarios para ascender de categoría, que mejor oportunidad con este programa, en la cual también nos comunicaron de acuerdo al número de profesores nos iban a dar una computadora. Fue así que tenemos una bonita sala de computación. La gente que tiene a cargo este proyecto es una gente preparada, nos han brindado amistad, confianza, conocimiento, nos llama a seminarios que son favorables".

\section{Director caso 1.}

El comentario anterior, nos muestra que la capacitación es bienvenida y acogida por parte del profesorado. Aunque, la visión de la misma se limita a que la capacitación les sirve para "ascender de categoría" en el magisterio, quedando inconsistente la finalidad misma de la capacitación: la motivación de adquirir competencias para un desarrollo profesional con respecto al uso pedagógico de las TIC en la enseñanza.

En otro de los casos, la capacitación adquirida se desvela del siguiente modo:

"Esta capacitación que hemos recibido nos ayuda a prepararnos. Siempre y cuando nos capaciten y tengamos la ayuda suficiente para que cada maestro tenga un computador en su clase".

Maestra del caso 1. 
- La formación es recibida y asimilada dependiendo del interés y la motivación que cada docente tenga. Para unos la capacitación a priori, serviría para tener méritos; para otros, serviría para mejorar su clase y por consiguiente el aprendizaje de sus alumnos.

\section{"La capacitación no sólo sirve para nosotros, sino también porque así estamos preparados para ayudar a nuestros alumnos".}

\section{Maestra del caso 2.}

- Podemos finalizar este apartado, comentando que formar parte del proyecto "De tal palo, tal astilla", ha supuesto una mejora de la imagen de la institución tanto para los padres como para la comunidad educativa.

"Con este proyecto, hemos ganado una buena reputación en la comunidad educativa, nuestra escuela tiene un alto número de alumnos en este curso escolar"

Director caso 2.

\section{Dimensión 3: Enseñanza con TIC}

- En las aulas observadas los alumnos disponen de un ordenador. El modelo de agrupamiento para el trabajo con los ordenadores es por parejas y de manera colaborativa. Creemos que es una de las estrategias privilegiadas en los nuevos ambientes de aprendizaje apoyados con las TIC (Peñaherrera, 2008 b).

- Los niños trabajan en el aula de informática por la mañana con su respectivo profesor, normalmente tienen su horario de clase, de acuerdo a los paralelos o cursos, 45 minutos trabaja cada curso.

- La mayor parte de las actividades se la realizan a través del aprendizaje basado en proyectos. Esta metodología representa una forma de trabajo autónoma, lo cual permite pasar de la memorización a la exploración, de la reflexión a la acción (Peñaherrera, 2008 b).

- Los profesores plasman los proyectos de aula de un contenido curricular en plan de clase. En uno de los casos observados, las TIC son registradas en esa programación de manera instrumental y son utilizadas como un refuerzo o como afianzamiento del aprendizaje, más no son integradas en un proceso amplio y pedagógico intencionado. Existe un desequilibrio pedagogía vs tecnología.

- Otro aspecto que nos ha llamado la atención es que en el Programa Curricular Insitucional, en el momento de la investigación, no se registra las TIC por ningún lado, ni en los objetivos, ni en los contenidos, ni tan siquiera como recursos. Esto nos indica, la carencia de una adecuada planificación global y que desde la política propia del centro no se encause su integración en armonía con las necesidades e intereses de la comunidad educativa. 
- Por otro lado, en ninguno de los casos observados se han encontrado materiales adaptados al alumnado con Necesidades Educativas Especiales (NEE), como es el caso de alumnos con dificultades de aprendizaje. Creemos que desde un punto de vista psicopedagógico, es de vital importancia hacer adaptaciones curriculares y materiales para estos alumnos.

\section{Dimensión 4: Aprendizaje con TIC}

- En relación al aprendizaje con TIC, los alumnos están altamente motivados con la utilización de las TIC. Se han familiarizado muy rápido con los ambientes informáticos y el contacto con este entorno les permite tener otras alternativas de aprender. La peculiaridad de combinar texto, sonido e imagen les abre nuevas posibilidades.

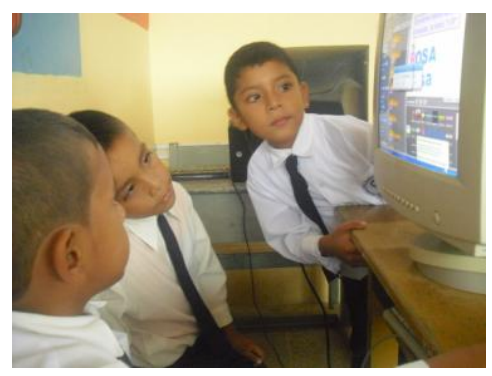

Foto 1. Alumnado participante del proyecto.

- Sin embargo, el alumnado puede perder esa motivación debido a que es el profesor quién decide cuándo utilizarlo. Por ejemplo, una profesora después de un prolongado tiempo (alrededor de 50 minutos) les "permite" a los niños utilizar los ordenadores. Desde el inicio, éstos han estado apagados, mientras los niños hacían distintos tipos de comentarios como: "señorita juguemos con la computadora", después otro le insiste "señorita a la computadora..." y así hasta que ella presionada comenta "si se portan mal no van a las computadoras". Es decir, la profesora asocia la computadora como una "recompensa" o "castigo" y consecuentemente los alumnos modifican ese comportamiento, hasta que la conducta de los niños deja de persistir.

Esta observación es corroborada por la misma profesora:

"Ha habido un cambio, ellos les encanta la computadora, es lo que les llama la atención. Pero ellos, tienen que aprender a escuchar la orden. Ahh... si nosotros no nos portamos bien no podemos trabajar con la computadora...

Maestra del caso 3.

Finalmente, las TIC se las utiliza después del recreo. Podríamos decir que los niños son espectadores del proceso de enseñanza y sujetos pasivos de las TIC.

- Los proyectos de aula realizados son expuestos en una "jornada de puertas abierta". Estas jornadas constituyen un elemento de evaluación y balance del proyecto anualmente. 


\section{ALGUNOS ELEMENTOS CONCLUYENTES Y DE PROSPECTIVA}

- Existen algunos aspectos críticos en la implementación de programas, sobre todo relacionado a la evaluación del uso de las TIC en proyectos y programas. Estos "nudos" aún persisten, la ausencia de una política correlacionada entre el nivel ejecutor (escuela) y el nivel diseñador (Gobierno) afecta el modo de integración de las TIC. Por un lado, se encuentran las políticas que desde los gobiernos se diseña, y por otro, la realidad escolar (liderazgo para la introducción de las TIC en el aula, ausencia de tener claro por qué se quiere utilizar el ordenador y sus ambientes de aprendizaje en clase, etc.) aún dista de lo que realmente se quiere lograr.

- La integración de las TIC no es una tara fácil, depende de muchos niveles, decisiones y agentes. Lo que sí es importante destacar, en nuestra investigación, es que todos los centros educativos participantes del proyecto difícilmente podrían haber iniciado el uso de las TIC en sus prácticas educativas, estas son consecuencia del impulso "De Tal Palo, Tal Astilla".

- Los docentes están conscientes del cambio y se muestran receptivos ante la integración de la tecnología en el aula. Sin embargo, esto no es suficiente, se necesita un verdadero liderazgo de los directivos y de iniciativa por parte del profesorado.

- Por otro lado, creemos que el tiempo que se le dedican a la utilización de los programas informáticos es insuficiente y los programas como Scracht necesitan por sus características, mucho más tiempo, la sola conclusión de una actividad puede tomar varias clases. Se debe tener en cuenta que la organización del conocimiento en un currículum disciplinar exige el tratamiento de muchos temas abordados de manera aislada que deben ser distribuidos temporalmente durante el curso escolar.

- Hemos de comentar que la integración de las TIC en el aula debe ser asumido con sentido de pertenencia por un número significativo de profesores, sin este indicador los cambios van hacer pocos relevantes y las prácticas pedagógicas con TIC serán poco significativas.

- Por tanto, el uso de los ordenadores y demás tecnologías digitales, por lo general (salvando ciertas peculiaridades) no se traducen en un replanteamiento significativo y radical del enfoque de enseñanza-aprendizaje que están utilizando y de algunos de los elementos de la programación de aula.

- Las TIC se incorporan como un complemento adhoc a la metodología habitual del profesorado. Adaptándose en mayor o menor medida Al modelo pedagógico del profesorado.

- No basta con poner equipos en las escuelas, y tampoco con enseñar a los docentes a usar de manera instrumental el ordenador, todo ello necesario pero no suficiente. Mientras la estructura y organización de la escuela siga favoreciendo propuestas centradas en el docente o en los materiales de aprendizaje más que en los estudiantes; un conocimiento representado como algo dado y externo al alumnado y no como algo que deviene y que el alumno tiene que construir, los ordenadores seguirán siendo una innovación tecnológica pero faltará mucho para que se conviertan en una mejora o innovación educativa (Mcclintock, 2000). 
- Finalmente, hemos de indicar que la integración de las TIC es un proceso a largo plazo y como hemos visto depende de algunos aspectos. Además, requiere de algunas condiciones para lograr apropiarse pedagógicamente de ellas (programación curricular institucional, cambios de estilos de enseñanza, formar parte de redes profesionales, etc.); Todo hace pensar que no basta con poner equipos en las escuelas, y tampoco con enseñar a los docentes a usar de manera instrumental el ordenador, todo ello necesario pero no suficiente. Mientras la estructura y organización de la escuela siga favoreciendo propuestas centradas en el docente o en los materiales de aprendizaje más que en los estudiantes; un conocimiento representado como algo dado y externo al alumnado y no como algo que deviene y que el alumno tiene que construir, los ordenadores seguirán siendo una innovación tecnológica pero faltará mucho para que se conviertan en una mejora o innovación educativa (Mcclintock, 2000).

- Si bien los casos estudiados no es susceptible de generalización, si lo es de generalización naturalista, una generalización que nace a partir del análisis que cada lector hace de él (Stake, 1994), es el lector quien generaliza, desde mi punto de vista, un tipo de generalización más valiosa, más que nada por el tipo de reflexión que es capaz de suscitar.

- Finalmente, si bien es cierto, el trabajo que viene realizando el gobierno ecuatoriano en conjunto con las universidades, en este caso, la ESPOL, es un buen inicio para que las TIC se integren gradualmente en las escuelas, también es cierto, que mientras el profesorado no emplee un modelo pedagógico en coherencia con las TIC, las nuevas tecnologías difícilmente podrán formar parte de la cultura de innovación y del quehacer docente.

\section{REFERENCIAS}

AREA, M. (2005 a). Tecnologías de la información y comunicación en el sistema escolar. Una revisión de las líneas de investigación. Relieve, 11 (1), 3-25. Recuperado el 14/01/2012. http://www.uv.es/RELIEVE/v11n1/RELIEVEV11n1_1.htm

AREA, M. (2010 b). El proceso de integración y uso de pedagógico de las TIC en los centros educativos. Un estudio de casos. Revista de Educación, 352, 77-97.

ADELL, J. (2011). El desarrollo profesional docente y las TIC. [Documento en línea]. Recuperado el 14 de enero de 2012http://es.scribd.com/doc/26306552/El-DesarrolloProfesional-del-Docente-y-las-TIC.

BECKER, H.J (2000). Findings from Teaching, Learning, and Computing Survey: Is Larry Cuban Right? Recuperado el 14 de enero de 2012 http://www.crito.uci.edu/tlc/html/findings.html

BALANSKAT, A. BLAMIRE, R. y KEFALA, S. (2006). The ICT impact report: A review of studies of ICT impact on schools in Europe. [Documento en línea]. Recuperado el 14 de enero de 2012http://ec.europa.eu/education/doc/reports/doc/ictimpact.pdf. 
CABERO, J.; LLORENTE, M.C. (2010). "Comunidades virtuales para el aprendizaje". [Artículo en línea]. EDUTEC, Revista Electrónica de Tecnología Educativa. Núm. 34 /Diciembre 2010. Recuperado el 14 de enero de 2012 http://edutec.rediris.es/revelec2/revelec34/

CUMBRE MUNDIAL SOBRE LA SOCIEDAD DE LA INFORMACIÓN. (2003). Agenda de solidaridad mundial. Recuperado 14 de enero de 2012 en file://localhost/http http/::www.itu.int:wsis:outcome:booklet:plan action D-es.html14 de enero de 2012.

CUMBRE MUNDIAL SOBRE LA SOCIEDAD DE LA INFORMACIÓN. (2005). Informe Compromiso de Túnez. Recuperado 14 de enero de 2012 http://www.itu.int/wsis/documents/index2es.html

CONATEL (2006). Libro Blanco. Estrategia para el desarrollo de la sociedad de la información en el Ecuador. Recuperado 14 de enero de 2012 http://www.conatel.gov.ec/website/conectividad/sociedad.php?cod cont=280

CONDIE, R y MUNRO, B. (2007). The Impact of ICT in Schools: a landscape review. UK: Becta.

COX, M. y WEBB, J. (2004). ICT and attainment: A review of the research literature ICT in Schools Research and Evaluation Series - No.17. Recuperado 14 de enero de 2012 http://www.becta.org.uk/page documents/research/ict attainment summary.pdf

COX, M. (2003). ICT and attainment: A review of the research literature ICT in Schools Research and Evaluation Series - No.17. DfES-Becta. Recuperado 14 de enero de 2012.

http://www.becta.org.uk/page documents/research/ict attainment summary.pdfCLARO, M. (2010). La incorporación de tecnologías digitales en educación. Modelos de identificación de buenas prácticas. Chile: Naciones Unidas.

GOETZ, J.P. y LECOMPTE, M.D. (1988). Etnografía y diseño cualitativo en investigación educativa. Madrid: Morata.

HUBERMAN, A. M. y MILES, M. B. (1994). Qualitative data analysis (2nd ed.) Thousand Oaks, CA: SAGE Publications.

KAGEL, M.M. (2003). Estudio de los cambios organizacionales producidos por un proyecto de informatización desarrollado en el centro escolar. Málaga: Facultad de Ciencias de la Educación, tesis doctoral inédita.

LAGOS, M. y SILVA, J. (2011). Estado de las experiencias 1 a 1 en Iberoamérica. Revista iberoamericana de educación. 56, 75-94. Recuperado 14 de enero de 2012. En http://www.rieoei.org/rie56a03.pdf

ESPOL-CTI. Informe de actividades octubre 2009 a Marzo 2010 "Mejoramiento de la Calidad de la Educación Pública en la Provincia de El Oro - Cantones Huaquillas, Arenillas, Las Lajas y Santa Rosa y las Tecnologías de Información para Fortalecer el Aprendizaje". Como autor Peláez, E. (2010). Ecuador.

MERRIAN, S. B. (1988). Case study research in education: A qualitative study. San Francisco: Jossey-Bass. 
MINISTERIO DE EDUCACIÓN DE ECUADOR. Informe Rendición de Cuentas 2010. Recuperado 10 de febrero de 2012 En http://www.educacion.gob.ec/ upload/Rendicion_2010.pdf

MINISTERIO DE EDUCACIÓN DE ECUADOR. Propuesta de estándares educativos. Recuperado 10 de febrero de 2012. En http http://www.educacion.gob.ec/generalidades-pes.html

MCCLINTOCK, R. (2000). Prácticas Pedagógicas Emergentes. El papel de las Tecnologías de la Información y de la Comunicación. Cuadernos de Pedagogía, 290, pp. 74-77

McFARLANCE, A. (2000). Establishing the Relationship between Networked Technology and Attainment: Preliminary Study 1. Recuperado 10 de febrero de 2012. En http://dera.ioe.ac.uk/1579/1/becta 2002 ImpaCT2 prelim report.pdf

PATTON, M. (1990). Qualitative evaluation and research methods. Newbury Park, CA: SAGE Publications.

PEÑAHERRERA, M. (2011 a). Evaluación de un programa de fortalecimiento del aprendizaje basado en el uso de las TIC en el contexto ecuatoriano. Revista Iberoamericana de Evaluación Educativa, 4(2), pp. 72-91. Recuperado el 14 enero 2012. http://www.rinace.net/riee/numeros/vol4num2/art4.pdf.PEÑAHERRERA, M. (2008 b). E-Culturas. Diseño, aplicación y evaluación de un programa de educación intercultural. Tesis doctoral no publicada. Universidad de Jaén. Jaén.

UNESCO. (2004). Las tecnologías de la información y la comunicación en la formación docente. París: UNESCO.

YIN, R. (1994). Case study research: Design and methods (2nd ed.). Thousand Oaks, CA. http://unesdoc.unesco.org/images/0012/001295/129533s.pdf (12 de julio del 2011)

SALOMÉ, T. (2010). Uso de TIC en la práctica docente de los maestros de educación básica y bachillerato de la ciudad de Loja. EDUTEC, Revista Electrónica de Tecnología Educativa. Núm. 33/Septiembre 2010. Recuperado el 20 de febrero de 2012. En http://edutec.rediris.es/revelec2/revelec33/

\section{Para citar este artículo:}

PEÑAHERRERA, M. (2012).Uso de TIC en escuelas públicas de Ecuador: Análisis, reflexiones y valoraciones. EDUTEC, Revista Electrónica de Tecnología Educativa, 40. Recuperado el dd/mm/aa de http://edutec.rediris.es/Revelec2/Revelec40/uso TIC escuelas publicas Ecuador analisis reflexion es valoraciones.html 